\title{
Residual disease in lymph nodes has no influence on survival in patients with incidental gallbladder cancer - institution experience with literature review
}

\author{
Mihajlo Dokic ${ }^{1,2}$, Urban Stupan', Sabina Licen ${ }^{3}$, Blaz Trotovsek ${ }^{1,2}$ \\ ${ }^{1}$ Division of Hepatobiliary Surgery and Liver Transplantation, Department of Abdominal Surgery, University Medical Center \\ Ljubljana, Ljubljana, Slovenia \\ 2 Faculty of Medicine, University of Ljubljana, Ljubljana, Slovenia \\ ${ }^{3}$ Nursing Department, Faculty of Health Sciences, University of Primorska, Izola, Slovenia
}

Radiol Oncol 2022; 56(2): 208-215.

Received 25 August 2021

Accepted 27 October 2021

Correspondence to: Prof. Blaž Trotovšek, M.D., Ph.D., Department of Abdominal Surgery, University Medical Center Ljubljana, Zaloška cesta 7, 1525 Ljubljana, Slovenia. E-mail: blaz.trotovsek@kclj.si

Disclosure: No potential conflicts of interest were disclosed.

This is an open access article under the CC BY-NC-ND license (http://creativecommons.org/licenses/by-nc-nd/4.0/).

Background. A better appreciation of the course and factors that influence incidental gallbladder cancer (iGBC) is needed to develop treatment strategies aimed to improve outcomes. The purpose of the study was to determine the influence of residual disease in the liver and lymph nodes on overall survival in re-resected patients with iGBC.

Patients and methods. Patients undergoing radical re-resection for iGBC from January 2012 to December 2018 were retrospectively identified. Patients with a 5 -year follow-up and submitted to complete resection with stage I, II and III disease were analysed. The influence of residual disease (RD) in liver and lymph nodes on survival was assessed using the Kaplan-Meier curves. In addition, the rest of the group was assessed based on type of primary/secondary procedure, number of harvested lymph nodes and RD in liver and/or lymph nodes.

Results. In this retrospective study 48 out of 58 (83\%) patients underwent re-resection. Among the group with a 5-year follow-up (re-operation between 2012-2014), 22 patients out of 28 (79\%) were re-resected. Survival analysis showed that patients with no RD in the liver and lymph nodes had statistically significant better 5-year survival than those with RD. Comparing 5-year survival rate in patients with RD in the liver or lymph nodes against no RD group, patients with $\mathrm{RD}$ in the liver had statistically significantly worse 5-year survival, while lymph node metastases did not show any statistically significant different in 5-year survival. Besides, a statistically significant better prognosis was found in stage II disease compared to stage III, as expected.

Conclusions. The most important predictors of a 5 -year survival in our study were RD in liver and stage of the disease. Lymph node metastases did not have any impact on the overall 5-year survival rate.

Key words: gallbladder; cancer; incidental; surgery; survival

\section{Introduction}

Gallbladder cancer (GBC) is a relatively uncommon aggressive malignancy, with an incidence of 2,5 per 100000 inhabitants in the United States of
America. ${ }^{1}$ It has a poor prognosis, the 5 -year survival rate is $5-15 \% .^{2}$ The only curative treatment option is $\mathrm{R} 0$ resection of the disease. ${ }^{3}$

Usually, patients with GBC are discovered incidentally with ultrasound (US) or computed to- 
mography (CT) because of unspecific symptoms or incidentally during the laparoscopic cholecystectomy. ${ }^{4} \mathrm{CT}$ is the best diagnostic tool to determine the resectability of such patients. ${ }^{5}$ Staging accuracy of CT may be augmented by contrast-enhanced magnetic resonance imaging (MR), which can provide a more detailed evaluation of the hepatic ducts. ${ }^{4}$ In patients with suspected or known GBC, 18-FDG positron emission tomography (PET)-CT has demonstrated the ability to detect occult metastases with a sensitivity of $56 \%{ }^{6}$

GBC most commonly spreads from the gallbladder to the pancreaticoduodenal and hepatic artery lymph nodes (LN), which are considered as regional lymph nodes. Spread to the coeliac and aortocaval lymph nodes represents metastatic disease. Detection of clinically occult metastasis in patients with GBC is difficult to achieve preoperatively due to aggressiveness of the disease and is a contraindication for radical surgical approach. ${ }^{7,8}$ If the imaging findings are suspicious of advanced disease with peritoneal spread or distant nodal involvement, diagnostic laparoscopy or laparotomy should be performed before re-resection. ${ }^{9}$

According to the recent perspective, minimally invasive surgery in oncologic GBC resection is not recommended. ${ }^{3}$ Oncological principles of adequate pancreaticoduodenal and hepatic artery lymphadenectomy and R0 liver margins are the standard of care. $^{3}$

Incidentally discovered GBC after cholecystectomy presents a diagnostic and therapeutic challenge. Incidental GBC (iGBC) is reported in approximately $0.7 \%$ of patients undergoing cholecystectomy. Ability to gather additional staging information during workup is beneficial but the extent of disease and spread after first procedure is often not detectable. The overall incidence of the residual disease (RD) varies according to T-stage of the primary tumour. In T1 tumours can be as high as $37.5 \%, 56.7 \%$ in T2 tumours, and $77.3 \%$ in T3 tumours. ${ }^{10}$ Incidence of RD in the liver bed and/ or LNs is lower, ranging from $12 \%$ in patients with $\mathrm{T} 1$ tumours to $46 \%$ in those with T3 tumours. ${ }^{10}$ The general opinion is that patients need to be reresected if $\mathrm{R} 0$ margins can be accomplished, but there is still some debate which patients benefit the most. ${ }^{11-15}$ Re-resection should be performed early after the primary procedure but after thorough diagnostic workup to exclude the patients who would not benefit from radical surgery. ${ }^{3}$

In preoperative workup CT or MRI is necessary but the role of PET-CT is unknown, since there is always inflammation around the operative field, which can lead to misconclusions. Thus, the role of PET-CT in iGBC before re-resection surgery remains undetermined, but remains additional diagnostic tool with small impact on decision making (13\% of patients), probably useful for finding distant metastases and when questionable or concerning features are apparent on CT or MRI. ${ }^{10}$

Operative strategy in re-resection consists of staging laparoscopy, liver resection and lymphadenectomy. ${ }^{3}$ Prior to performing laparotomy at the time of re-resection, a staging laparoscopy may be performed to evaluate distant metastases. ${ }^{16}$

Port site resection is not supported by the literature. ${ }^{3}$ It is not associated with improved survival and was associated with higher complication rate such as incisional hernia (15\%). ${ }^{12}$ Positive histology of port sites is associated with the peritoneal dissemination of primary tumour and has a poor prognosis. ${ }^{17}$ Therefore routine port site resection is not indicated.

LN dissection can be limited to the hepatoduodenal ligament (regional LN), as extended LN excision (coeliac and aortocaval) is not associated with improved outcomes because the involvement of these nodes represents distant metastatic disease. ${ }^{9,18}$ The incidence of $\mathrm{LN}$ involvement varies by T-stage, $12 \%, 31 \%$ and $45 \%$ in patients with $\mathrm{T} 1 \mathrm{~b}, \mathrm{~T} 2$ and T3 tumours, respectively. ${ }^{10}$ Lymphadenectomy and radical liver resection were associated with improved survival in comparison with radical liver resection alone in patients with $\mathrm{T} 1 \mathrm{~b}$ and $\mathrm{T} 2$ tumours. ${ }^{19}$ Biopsy of aortocaval LN should be performed routinely at the initiation of the procedure and may be used to tailor surgical approaches. ${ }^{18,20,21}$

Resection with negative margins is mandatory. $3,7,10,12,13,22$ Major hepatectomy compared with wedge resection ( $3 \mathrm{~cm}$ margin) or a formal segment $4 b-5$ resection has not been associated with improved survival, but had a higher complication rate. ${ }^{22-24}$

Routine bile duct resection has been shown repeatedly to have no impact on improved survival but increases morbidity. 10,22 Furthermore, it is associated with regional LN involvement and has a poor prognosis. ${ }^{24}$ It is indicated in patients with positive cystic duct margin after cholecystectomy or when it is necessary during re-resection for the achievement of negative margins. ${ }^{3}$

Timing of re-resection is still a controversial issue. When GBC is discovered during the primary procedure (during laparoscopy or classic cholecystectomy) surgeon should either continue with definitive surgery with the liver resection and lymphadenectomy or should stop the procedure and 
transfer such patient into the HPB centre. If it is discovered due to pathologic findings, then intensive workup for staging is necessary. The optimal time interval for re-resection for incidentally discovered gallbladder cancer appears to be between 4 and 8 weeks after the initial cholecystectomy. ${ }^{25}$ Time to re-resection was not confirmed as a predictor of survival in a study of Fuks et al.. ${ }^{12}$

This retrospective study has been performed to evaluate the conditional survival rate at multiple time points in patients with RD in liver and LN after re-resection for iGBC.

\section{Patients and methods}

This study was designed as retrospective. All patients with iGBC referred to the Department of Abdominal Surgery of the University Medical Centre Ljubljana from January 2012 to December 2018 were included in a study. Survival analysis of those patients with a 5-year follow up (re-resection between 2012-2014) was performed. Patients were identified from a prospectively maintained computer database in 2019.

This study was approved by the Slovenian National Medical Ethics committee (number 0120514/2019/4). Study also conforms to Declaration of Helsinki and European Medicines Agency Guidelines for Good Clinical Practice.

All patients were presented at the multidisciplinary meeting (MDM) before the re-resection. Preoperative assessment included CT of the abdomen and pelvis. Besides, a review of the histopathology of the gallbladder specimen removed during primary surgery (laparoscopic or open cholecystectomy) was performed to determine the histologic type, routine blood count, and CA19-9. In patients (pT1b and more) with non-metastatic, locally resectable disease was offered surgical exploration with an intent to perform a radical re-resection. Data collected included patient demographics, pathological status, type of further surgical procedure, time between the primary cholecystectomy and the radical re-resection, and overall survival $(1,3$, and 5 years). Postoperatively, patients were followed up at 3-monthly intervals for the first 2 years, then every 6 months for another two years and then yearly.

Continuous data are presented as median and range. Overall survival analysis was undertaken using the Kaplan - Meier method. Survival curves were compared using the log rank test. $P$ values of $<0.05$ were considered significant. Statistical analy- sis was performed using SPSS for MacOS, 25th edition.

\section{Results}

Of 58 patients, 48 (42 female and 6 male patients) median age 73 years (range 37-85 years) underwent radical re-resection. Only exploration was performed in $17 \%(10 / 58)$ of the patients. $83 \%$ $(48 / 58)$ of the patients were treated with re-resection. Among the 28 analysed patients 79\% (22/28) were re-resected.

\section{Primary procedure}

Laparoscopic cholecystectomy was performed in 42 out of 58 patients with a conversion rate of $19 \%$. Classic cholecystectomy was performed in 16 patients (28\%). Patients had tumour stage T1b, T2 and $\mathrm{T} 3$ in $7 \%, 53 \%$ and $24 \%$ respectively. At least one LN was harvested in $70 \%$ and was positive in $13 \%$ of patients. Four patients had pT3 tumour and 4 patients had pT2 tumour and positive LN on histological examination after the primary procedure. Six or more lymph nodes were harvested in 27 out of 48 patients.

\section{Preoperative workup}

CT was performed in all 58 patients and residual disease was present at $17 \%$ of them. In $3(5 \%)$ patients the residual disease was found in liver, $3(5 \%)$ patients had a residual disease in $\mathrm{LN}$ and $1(2 \%)$ had residual disease both in liver and LN. Diagnosis/staging made by CT was correct in $71 \%$ of patients, comparing CT staging and histopathology. In $27 \%$ of patients CT underestimated stage of the disease and in $2 \%$ of patients CT overestimated the extent of disease.

\section{Re-resection}

In 48 out of 58 patients who underwent exploratory laparotomy, re-resection was performed. In $90 \%$ (43/48) of them R0 resection was achieved, in $10 \%$ of them, R1 resection was achieved.

The median duration between two surgeries was 49 days (range 10-180). Exploratory laparotomy only was performed in $10(17 \%)$ patients. Reasons for exploration only were distant metastases in 8 patients and local unresectability in 2 patients.

28 out of 48 (58\%) patients who had R0 resection underwent resection of segment $4 \mathrm{~b}-5$ and lym- 
TABLE 1. Characteristics of patients with incidentally detected gallbladder cancer at the time of second surgery and survival characteristics at 1,3 , and 5 years after the intervention

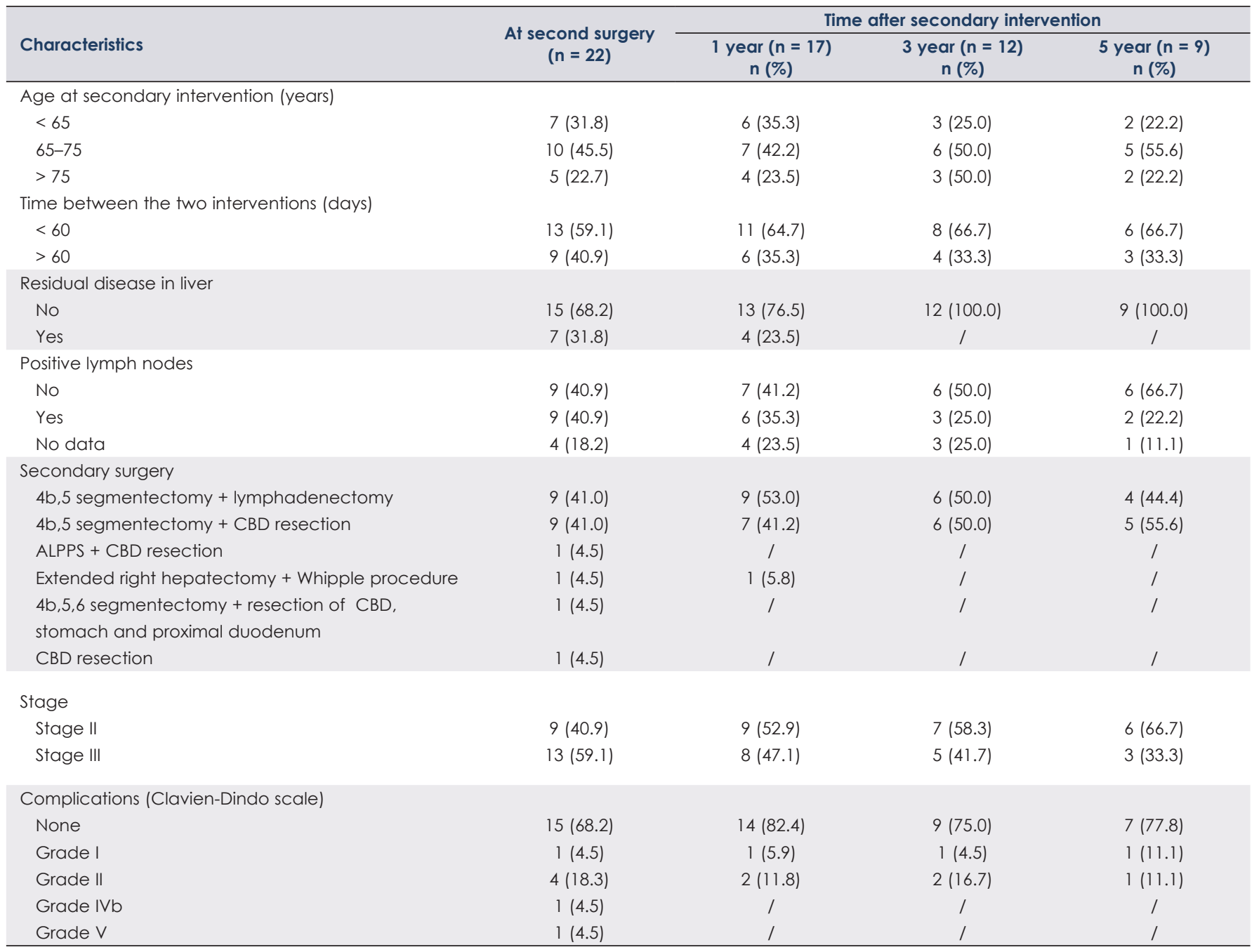

ALPPS = associating liver partition and portal vein ligation for staged hepatectomy; $C B D=$ common bile duct

phadenectomy. In 14 (29\%) patients' additional resection of the common bile duct was performed due to cystic duct involvement. Right hemihepatectomy in 3 cases, right trisectionectomy in 3 cases one of them with additional cephalic pancreatoduodenectomy, was performed due to extent of the disease.

At our Institution lymphadenectomy of the hepatoduodenal ligament is mandatory in patients with GBC, therefore it was performed in all 48 patients. Extended lymphadenectomy of the celiac trunk and of aortocaval $\mathrm{LN}$ was performed in $45 \%$. In average 7,7 LN (range 2-35) was harvested during re-resection and 8,2 LN (range 2-32) in the analysed group. Six or more LN was harvested in 27 out of $48(56 \%)$ patients during re-resection overall and in 12 out of $28(43 \%)$ patients in the analysed group.

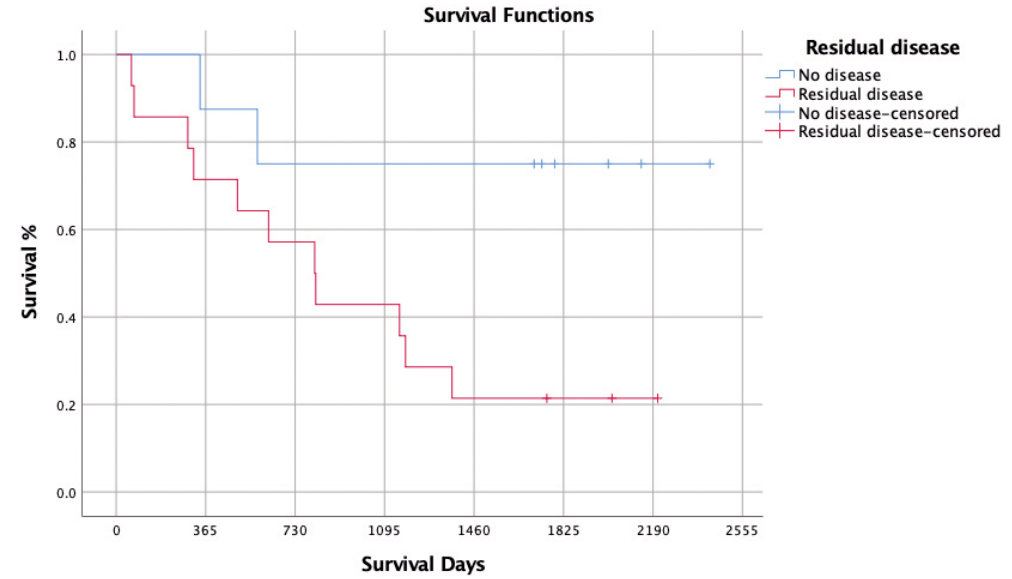

FIGURE 1. Kaplan-Meier comparing patients identified with or without residual disease following second surgery. 

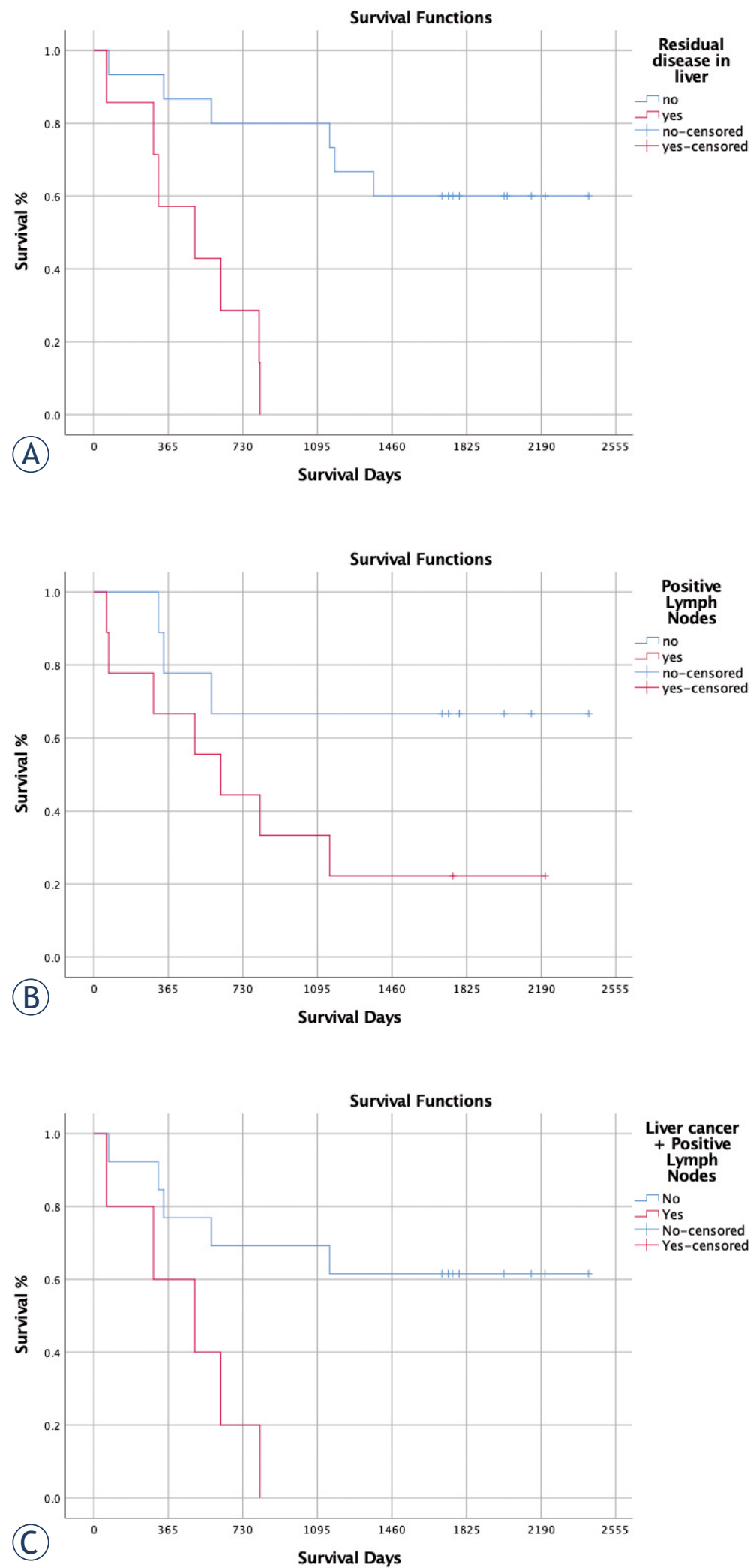

FIGURE 2. Kaplan-Meier comparing patients identified with or without residual disease in liver and positive lymph nodes following second surgery. (A) Residual disease in liver, (B) Positive Lymph Nodes), (C) Residual disease in liver and positive lymph nodes.
Resection achieved complete tumour removal in $36(75 \%)$ patients who underwent the "intention to treat" procedure. After final pathology patients were classified according to TNM $7^{\text {th }}$ ed. classification system. There were 4 patients in stage I, 21 in stage II, 21 in stage III and in stage IV 12 patients. Of those patients, all with stage I and II, 20/21 (95\%) with stage III disease and 3/12 (25\%) with stage IV disease underwent re-resection. Among the analysed group there were $0(0 \%), 9(32 \%), 13(46 \%)$ and $6(22 \%)$ patients with stage I, II, III and IV disease respectively. No one with stage IV disease was re-resected, and all other patients (22) were reresected.

\section{Survival}

Between 2012 and 2014, twenty-two patients proceeded to a second surgical procedure following diagnosis of incidental gallbladder cancer after initial cholecystectomy. Table 1 shows the characteristic of the patient at the time of secondary intervention as well as 1, 3 and 5 years after diagnosis.

All of the patient, that were analysed, were females, with median age of 67 at the time of a second surgical procedure (range 47-81 years). Median time between initial cholecystectomy and second surgery was 51 days (range 22-780).

For the whole group (48 patients), morbidity rate was $25 \%$ and median hospitalization time was 7 days. Out of 48 patients 1 died (2\%) on the $59^{\text {th }}$ day due to the liver failure and sepsis. 11 patients had some kind of postoperative complications, most of them level 2 by Clavien-Dindo scale (CD). One patient had pulmonary embolism. In 2 patients' level 3 by CD complication had occurred, 1 with wound infection, requiring negative pressure assisted wound closure (level 3a) and one with perforation of transverse colon (level 3b) which required reoperation. Patient with colon perforation was on corticosteroid therapy due to active form of ulcerative colitis. In 1 patient level $4 \mathrm{~b}$ CD complication was developed, due to haemorrhage, which needed surgical haemostasis.

Patients with any presence of RD, either in the liver or as positive LN, or both after the second surgery had a median estimated survival of 986 days ( $95 \%$ CI, 597 to 1376 days) compared to the patients without the RD. Based on a log rank test, the results show that there were differences in the overall survival distribution between the patients without RD and those with any RD that were statistically significantly different, $\chi 2(1)=4.561, p=$ 0.033 (Figure 1). 
TABLE 2. Output of the multivariate Cox regression model

\begin{tabular}{|c|c|c|c|c|c|c|}
\hline \multicolumn{7}{|c|}{ Estimates } \\
\hline Variable & Beta & $\begin{array}{l}\text { Standard } \\
\text { error }\end{array}$ & $p$-value & $\begin{array}{c}\text { Hazard ratio } \\
\exp (\text { Beta) }\end{array}$ & $95 \%$ co & interval \\
\hline RD in liver or/and positive lymph nodes (Yes/No) & 1.253 & 0.792 & 0.114 & 0.286 & 0.061 & 1.349 \\
\hline Stage (II/III) & 1.716 & 1.130 & 0.129 & 0.180 & 0.020 & 1.648 \\
\hline Age & 0.027 & 0.033 & 0.415 & 1.028 & 0.962 & 1.097 \\
\hline Days between the two operations & 0.001 & 0.002 & 0.717 & 0.999 & 0.996 & 1.003 \\
\hline
\end{tabular}

$\mathrm{RD}=$ residual disease

Patients with the presence of RD in the liver and positive lymph nodes after the second surgery had a median estimate survival time of 457 days $(95 \%$ CI, 201 to 712 days) when compared with the patient without any of the RD. A log rank test was performed to determine whether there were differences in the survival distribution for patients with none $\mathrm{RD}$ and those with $\mathrm{RD}$ in liver and positive lymph nodes. The survival distributions between the two groups were statistically significantly different, $\chi 2(1)=6.807, p=0.009$ (Figure 2). In addition, patients with the presence of RD in liver or positive lymph nodes following second surgery had an estimated median survival of 487 days (95\% CI, 278 to 696 days) and 881 (95\% CI, 370 to 1391 days, respectively). A log rank test was run to determine if there were differences in the survival distribution between patients with RD in liver and those without. The survival distributions were statistically significantly different, $\chi 2(1)=13.505, p<0.0001$. When comparing those with and without positive lymph nodes, the survival distributions were not statistically significantly different $\chi 2(1)=2.992, p=0.084$ (Figure 2).

All patients involved in the study were in the stages II or III. Patients that were in the stage III of the disease had a median estimation survival time of 622 days (95\% CI 68 to 1176) days. A log rank was run to determine differences in the survival distribution for the both groups. The survival distributions for the stage 2 and stage 3 patients were statistically significantly different $\chi 2(1)=4.860, \mathrm{p}=$ 0.027 (Figure 3).

The survival distributions for the patients age groups $(<65,65-75,>75$ years) and the time between the two intervention $(<60,>60$ days) were not statistically significantly different $\chi 2(2)=0.445$, $p>0.05$ and $\chi 2(1)=0.472, p>0.005$, respectively.

Further, a multivariate Cox model was performed to describe the risk factors associated with a lower survival. Age, cancer stage, time between

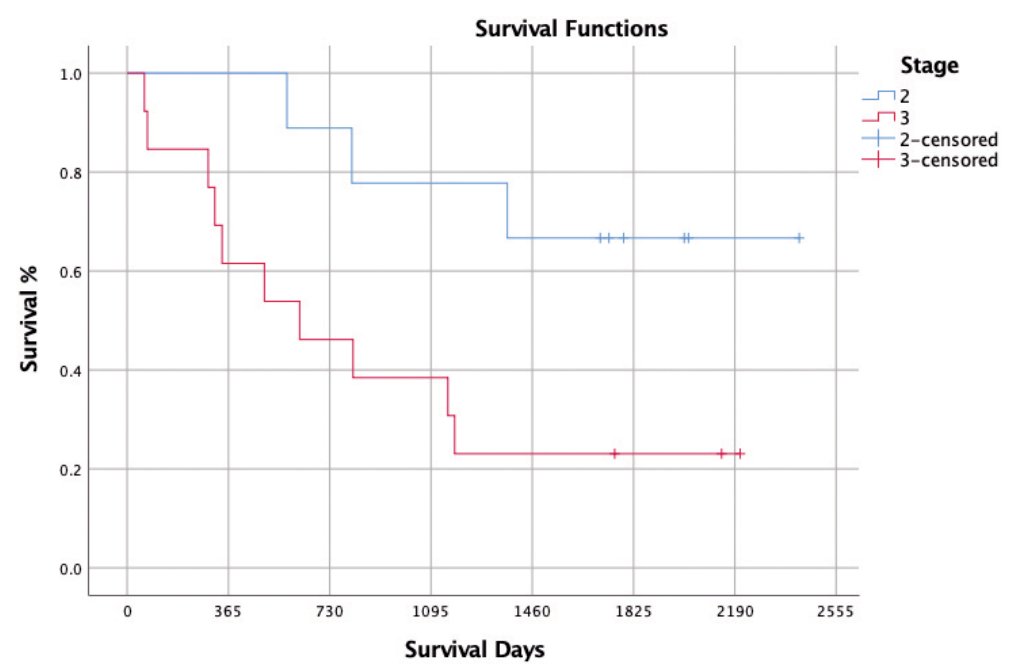

FIGURE 3. Kaplan-Meier comparing patients identified with II and III stage disease following second surgery.

both surgeries, RD in liver and positive lymph nodes, were included in the model. The Cox proportional hazards model demonstrated that RD in liver and/or positive lymph nodes $(p=0.114)$, TNM stage $(p=0.129)$, age $(p=0.415)$ and time between both the surgeries $(p=0.717)$ were not independent prognostic factors (Table 2).

\section{Discussion}

Cholecystectomy for gallstones is the second most frequent procedure in digestive surgery. iGBC is going to become an increasingly frequent medical entity since it can be found in one of every 150 cholecystectomies performed. ${ }^{10}$ Cholecystectomy is a treatment of choice and oncologically adequate for carcinoma in situ (Tis and T1a GBC). In the case of iGBC radical re-resection has been advocated for other stages of the disease as long as it is feasible. ${ }^{1,11-15}$ The aim of re-resection in incidental iGBC is definitive staging and when feasible the re- 
section of the entire tumour. ${ }^{2,3}$ Low mortality and morbidity in our study and better survival justifies re-resection. ${ }^{12} \mathrm{GBC}$ is more common in females ${ }^{12,26}$, in our study female to male ratio was 8:1 which is considerably higher than in published literature. With median age of 73 years we confirmed findings that $\mathrm{GBC}$ is more frequent in the elderly population. ${ }^{1,12,26,27}$ In the era of minimally invasive surgery, it is interesting that almost one-third of primary procedures - cholecystectomies, was performed through an open approach. ${ }^{28}$ One can only speculate that there were probably some changes of gallbladder wall on US that convinced the surgeon to use the open approach.

$\mathrm{CT}$ is the diagnostic modality of choice to determine resectability because of its capacity to interrogate portal nodes, peritoneal implants as well as vascular invasion. ${ }^{5}$ In cases of known GBC preoperative workup with CT or MRI is mandatory. Since there is always a sequel of the previous procedure, in the form of inflammation and/or fibrosis, imaging techniques may be misleading, especially PET CT. ${ }^{10}$ The sensitivity of CT in our study was $71 \%$ which is comparable with the data from the literature. ${ }^{10}$

Re-resection rate of $82 \%$ in our study is comparable with other published data ${ }^{12,16}$, where $18 \%$ of patients that were considered as resectable after preoperative workup becomes nonresectable during staging laparoscopy or exploratory laparotomy. Predictors of positive findings are T3 disease, a poorly differentiated tumour and positive margin at the time of original cholecystectomy. In these patients laparoscopy should always be performed before exploration..$^{16} \mathrm{R} 0$ resection was achieved in $90 \%$ of re-resected patients and in $75 \%$ in the whole group which is comparable with the data from the literature. ${ }^{12,16} 10 \%$ of patients with R1 resection had at least stage IIIb disease according to TNM $7^{\text {th }} \mathrm{ed}$. classification. Re-resection consisted of resection of liver segments $4 b, 5$ and lymphadenectomy in the majority of patients with additional resection of bile ducts in one third of them. More extensive reresections were performed in 6 fit patients all with stage IIIb or IV as a rescue procedure. In the literature radical resections that included hepatectomy, common bile duct (CBD) and/or vascular resection have been reported ${ }^{22,23}$, but without prolongation of overall survival. Increased morbidity and mortality associated with extended surgical procedures are an important factor in the survival and extended procedures should be reserved for medically fit patients after multidisciplinary discussion.

Lymphadenectomy and LN ratio has been shown to be an important predictor of survival af- ter surgery..$^{29}$ Positive regional LNs have an influence on survival in GBC. It is recommended that at least six LNs should be removed for accurate staging. ${ }^{30}$ In this study, an average of $7,7 \mathrm{LN}$ was procured at the re-resection for the whole group and $8,2 \mathrm{LN}$ for the analysed group. Underestimation of TNM stage before resection is a common finding. The residual tumour in the liver was found in 25\% of patients, and LN invasion in $29 \%$ of the patients. Review of re-resection specimen increased preoperative stage of the disease in $27 \%$ of our patients.

Although many surgeons advocate routine resection of the common bile duct at the time of curative resection ${ }^{10,12}$, there was no improvement in $\mathrm{R} 0$ resection rate in our study. CBD resection was performed in $30 \%$ and only 2 patients out of 14 had histologically proven infiltration of CBD. Therefore, authors conclude that routine resection of CBD is not recommended and should be done only when there is a strong suspicion of infiltration on previous histology, frozen section (positive margin at cystic duct), or CT. In the study of Araida et al. ${ }^{31}$, the resected and nonresected bile duct groups did not substantially differ in terms of the 5-year cumulative survival and local recurrence along the hepatoduodenal ligament. In our study additional resection of CBD was an important risk factor for postoperative morbidity as did other authors conclude. ${ }^{31}$

In this study, the most important predictor of survival is RD in liver. In the revised literature, $\mathrm{RD}$ at any anatomical location is associated with adverse prognosis ${ }^{1-3,26,27,32,33}$ as was also confirmed in this study. However, when comparing patients with RD in liver to the patients with no RD in liver, patients with RD in liver had worse 5-year survival. In the majority of literature, LN metastases are found to be the most important predictor of survival. ${ }^{1-3,26,27}$ When comparing patients with $\mathrm{RD}$ in LN to the patients with no RD in LN, no statistically significant differences in 5-year survival were found. Certainly, there are limitations because of a retrospective nature of the study and small sample group. Furthermore, the results may vary when we analyse the whole 48 patients. On the other hand, the average $\mathrm{LN}$ yield was 8,2 , which is significantly higher that the required 6 , which should be sufficient to properly evaluate influence of LN metastases on survival.

\section{Conclusions}

High rate of RD in liver bed and regional LN justifies re-resection in patients with GBC. It appears 
that re-resection is especially beneficial in patients with GBC stage T1b, T2 and T3. Some modifications of the surgical management in iGBC occurred during the years. Bisegmentectomy $4 b, 5$ or in some cases wedge resection is still highly recommended, but CBD resection is indicated only in selected cases. Regional lymphadenectomy with harvesting of at least $6 \mathrm{LN}$ is recommended. Aortocaval and celiac $\mathrm{LN}$ are harvested for staging at the beginning of the procedure and should be used for tailoring of it. The authors found that the most important predictor of survival is RD in liver bed not LN, therefore, reresection, should be considered whenever possible, knowing that it is essential to achieve R0 resection.

\section{References}

1. Goetze TO, Paolucci V. Benefits of reoperation of $T 2$ and more advanced incidental gallbladder carcinoma. Ann Surg 2008; 247: 104-8. doi: 10.1097/ SLA.0b013e318154bf5d

2. Sikora SS, Singh RK. Surgical strategies in patients with gallbladder cancer: nihilism to optimism. J Surg Oncol 2006; 93: 670-81. doi: 10.1002/jso.20535

3. Aloia TA, Járufe N, Javle M, Maithel SK, Roa JC, Adsay V, et al. Gallbladder Cancer: expert consensus statement. HPB (Oxford) 2015; 17: 681-90. doi: 10.1111/hpb.12444

4. Hueman MT, Vollmer CM, Pawlik TM. Evolving treatment strategies for gallbladder cancer. Ann Surg Oncol 2009; 16: 2101-15. doi: 10.1245/s10434009-0538-x

5. Li B, Xu XX, Du Y, Yang HF, Li Y, Zhang $Q$, et al. Computed tomography for assessing resectability of gallbladder carcinoma: a systematic review and meta-analysis. Clin Imaging 2013; 37: 327-33. doi: 10.1016/j.clinimag.2012.05.009

6. Rodríguez-Fernández A, Gómez-Río M, Medina-Benítez A, Moral J V-D, Ramos-Font C, Ramia-Angel JM, et al. Application of modern imaging methods in diagnosis of gallbladder cancer. J Surg Oncol 2006; 93: 650-64. doi: 10.1002/jso.20533

7. Butte JM, Redondo F, Waugh E, Meneses M, Pruzzo R, Parada $H$, et al. The role of PET-CT in patients with incidental gallbladder cancer. HPB (Oxford) 2009; 11: 585-91. doi: 10.1111/j.1477-2574.2009.00104.x

8. Kokudo N, Makuuchi M, Natori T, Sakamoto $Y$, Yamamoto J, Seki M, et al. Strategies for surgical treatment of gallbladder carcinoma based on information available before resection. Arch Surg 2003; 138: 741-50. doi: 10.1001/archsurg.138.7.741

9. Goere $D$, Wagholikar GD, Pessaux $P$, Carrère $N$, Sibert A, Vilgrain V, et al. Utility of staging laparoscopy in subsets of biliary cancers: laparoscopy is a powerful diagnostic tool in patients with intrahepatic and gallbladder carcinoma. Surg Endosc 2006; 20: 721-5. doi: 10.1007/s00464-005-0583-x

10. Pawlik TM, Gleisner AL, Vigano L, Kooby DA, Bauer TW, Frilling A, et al. Incidence of finding residual disease for incidental gallbladder carcinoma: implications for re-resection. J Gastrointest Surg 2007; 11: 1478-86; discussion 1486-7. doi: 10.1007/s11605-007-0309-6

11. Dixon E, Vollmer CM, Sahajpal A, Cattral M, Grant D, Doig C, et al. An aggressive surgical approach leads to improved survival in patients with gallbladder cancer: a 12-year study at a North American Center. Ann Surg 2005; 241: 385-94. Available at: http://www.ncbi.nlm.nih.gov/pubmed/15729060

12. Fuks $D$, Regimbeau JM, Le Treut $Y-P$, Bachellier P, Raventos A, Pruvot F-R, et al. Incidental gallbladder cancer by the AFC-GBC-2009 Study Group. World J Surg 2011; 35: 1887-97. doi: 10.1007/s00268-011-1134-3

13. Butte JM, Waugh E, Meneses M, Parada H, De La Fuente HA. Incidental gallbladder cancer: analysis of surgical findings and survival. I Surg Oncol 2010; 102: 620-5. doi: 10.1002/jso.21681

14. Hari DM, Howard JH, Leung AM, Chui CG, Sim M-S, Bilchik AJ. A 21-year analysis of stage I gallbladder carcinoma: is cholecystectomy alone adequate? HPB (Oxford) 2013; 15: 40-8. doi: 10.1111/j.1477-2574.2012.00559.x
15. Abramson MA, Pandharipande P, Ruan D, Gold JS, Whang EE. Radical resection for T1b gallbladder cancer: a decision analysis. HPB (Oxford) 2009; 11 656-63. doi: 10.1111/j.1477-2574.2009.00108.x

16. Butte JM, Gönen $M$, Allen PJ, D’Angelica MI, Kingham TP, Fong $Y$, et al. The role of laparoscopic staging in patients with incidental gallbladder cancer. HPB (Oxford) 2011; 13: 463-72. doi: 10.1111/j.1477-2574.2011.00325.x

17. Maker A V., Butte JM, Oxenberg J, Kuk D, Gonen M, Fong Y, et al. Is port site resection necessary in the surgical management of gallbladder cancer? Ann Surg Oncol 2012; 19: 409-17. doi:10.1245/s10434-011-1850-9

18. Kondo S, Nimura Y, Hayakawa N, Kamiya J, Nagino M, Uesaka K. Regional and para-aortic lymphadenectomy in radical surgery for advanced gallbladder carcinoma. Br J Surg 2000; 87: 418-22. doi: 10.1046/j.13652168.2000.01384.x

19. Jensen EH, Abraham A, Jarosek S, Habermann EB, Al-Refaie WB, Vickers $\mathrm{SA}$, et al. Lymph node evaluation is associated with improved survival after surgery for early stage gallbladder cancer. Surgery 2009; 146: 706-11. doi: 10.1016/j.surg.2009.06.056

20. Meng $H$, Wang $X$, Fong $Y$, Wang $Z H$, Wang $Y$, Zhang ZT. Outcomes of radical surgery for gallbladder cancer patients with lymphatic metastases. Jpn J Clin Oncol 2011; 41: 992-8. doi: 10.1093/jjco/hyr072

21. Shirai Y, Sakata J, Wakai T, Ohashi T, Ajioka Y, Hatakeyama K. Assessment of lymph node status in gallbladder cancer: location, number, or ratio of positive nodes. World J Surg Oncol 2012; 10: 87. doi: 10.1186/1477-7819-10-87

22. D'Angelica M, Dalal KM, DeMatteo RP, Fong Y, Blumgart LH, Jarnagin WR. Analysis of the extent of resection for adenocarcinoma of the gallbladder. Ann Surg Oncol 2009; 16: 806-16. doi: 10.1245/s10434-008-0189-3

23. Kurosaki I, Hatakeyama K, Minagawa M, Sato D. Portal vein resection in surgery for cancer of biliary tract and pancreas: special reference to the relationship between the surgical outcome and site of primary tumor. $J$ Gastrointest Surg 2008; 12: 907-18. doi: 10.1007/s11605-007-0387-5

24. Birnbaum DJ, Viganò L, Ferrero A, Langella S, Russolillo N, Capussotti L. Locally advanced gallbladder cancer: Which patients benefit from resection? Eur J Surg Oncol 2014; 40: 1008-15. doi: 10.1016/j.ejso.2013.10.014

25. Ethun CG, Postlewait LM, Le N, Pawlik TM, Buettner S, Poultsides G, et al. Association of optimal time interval to re-resection for incidental gallbladder cancer with overall survival: a multi-institution analysis from the US extrahepatic biliary malignancy consortium. JAMA Surg 2017; 152: 143-9. doi: 10.1001/jamasurg.2016.3642

26. Cavallaro A, Piccolo G, Panebianco V, Menzo EL, Berretta M, Zanghi A, et al. Incidental gallbladder cancer during laparoscopic cholecystectomy: managing an unexpected finding. World J Gastroenterol 2012; 18: 4019-27. doi: 10.3748/wjg.v18.i30.4019

27. Muratore A, Amisano M, Viganò L, Massucco P, Capussotti L. Gallbladder cancer invading the perimuscular connective tissue: results of reresection after prior non-curative operation. J Surg Oncol 2003; 83: 212-5. doi: 10.1002/jso.10258

28. Paolucci V, Neckell M, Götze T. Das „okkulte” Gallenblasenkarzinom - CAE-S/ CAMIC-Zentralregister. Zentralbl Chir 2003; 128: 309-12. doi: 10.1055/s2003-38795

29. Negi SS, Singh A, Chaudhary A. Lymph nodal involvement as prognostic factor in gallbladder cancer: location, count or ratio? J Gastrointest Surg 2011; 15: 1017-25. doi: 10.1007/s11605-011-1528-4

30. Ito H, Ito K, D'Angelica M, Gonen M, Klimstra D, Allen P, et al. Accurate staging for gallbladder cancer: implications for surgical therapy and pathological assessment. Ann Surg 2011; 254: 320-5. doi: 10.1097/ SLA.0b013e31822238d8

31. Araida T, Higuchi R, Hamano M, Kodera Y, Takeshita N, Ota T, et al. Should the extrahepatic bile duct be resected or preserved in RO radical surgery for advanced gallbladder carcinoma? Results of a Japanese Society of Biliary Surgery Survey: a multicenter study. Surg Today 2009; 39: 770-9. doi: 10.1007/s00595-009-3960-6

32. Gil L, de Aretxabala X, Lendoire J, Duek F, Hepp J, Imventarza O. Incidental gallbladder cancer: How residual disease affects outcome in two referral HPB centers from South America. World J Surg 2019; 43: 214-20. doi: 10.1007/s00268-018-4762-z

33. Butte JM, Kingham TP, Gönen M, D'Angelica MI, Allen PJ, Fong $Y$ et al. Residual disease predicts outcomes after definitive resection for incidental gallbladder cancer. J Am Coll Surg 2014; 219: 416-29. doi:1 0.1016/j.jamcollsurg.2014.01.069 